\title{
Lidil
}

Revue de linguistique et de didactique des langues

$59 \mid 2019$

Prononcer les langues : variations, émotions, médiations

\section{[tyblyty'ty/ləSapopwẽ'ty] (Turlututu, le chapeau pointu) ou de la perception à la production de la voyelle /y/ par des apprenants polonophones}

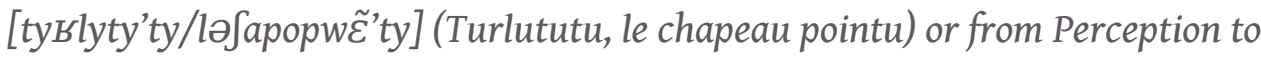
Production of the Vowel /y/ by Polish Speakers

\section{Dominique Hamm et Magdalena Dańko}

\section{OpenEdition}

\section{Journals}

Édition électronique

URL : http://journals.openedition.org/lidil/6201

DOI : 10.4000/lidil.6201

ISSN : 1960-6052

\section{Éditeur}

UGA Éditions/Université Grenoble Alpes

\section{Édition imprimée}

ISBN : 978-2-37747-090-7

ISSN : 1146-6480

\section{Référence électronique}

Dominique Hamm et Magdalena Dańko, « [tyъ lyty'ty/ləJapopwẽ'ty] (Turlututu, le chapeau pointu) ou de la perception à la production de la voyelle /y/ par des apprenants polonophones », Lidil [En ligne], 59 | 2019, mis en ligne le 01 mai 2019, consulté le 03 mai 2019. URL : http://journals.openedition.org/ lidil/6201 ; DOl : 10.4000/lidil.6201

Ce document a été généré automatiquement le 3 mai 2019.

(C) Lidil 


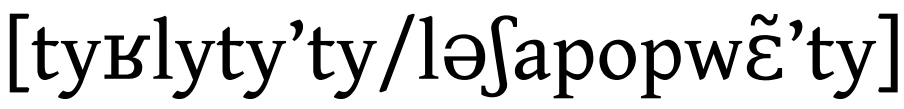 (Turlututu, le chapeau pointu) ou de la perception à la production de la voyelle /y/ par des apprenants polonophones}

[tyslyty'ty/ləSapopwẼ'ty] (Turlututu, le chapeau pointu) or from Perception to Production of the Vowel /y/ by Polish Speakers

Dominique Hamm et Magdalena Dańko

\section{Cadres théoriques de la recherche et objectifs visés}

1 Notre étude s'inscrit dans le cadre de la didactique de l'oral du français langue étrangère (FLE) et porte sur la perception/production de la parole d'apprenants. Notre arrière-plan théorique se fonde sur les modèles d'acquisition des sons en langue étrangère, notamment ceux de Polivanov (1931), Troubetzkoy (1939), Kuhl (1991), Best (1994) et Flege (1995).

2 Tout ce que nous entendons passe par une sorte de système de cribles décrit par Troubetzkoy (1939). Ainsi, tous les phonèmes nouveaux sont identifiés et catégorisés en fonction du système phonologique de la langue première des auditeurs (L1). Il en résulte que, dans le premier réflexe, inconsciemment, l'apprenant puise dans son système phonologique d'origine afin de trouver un son équivalent qui ressemblerait le plus au nouveau. Il s'agit, en effet, d'une prédisposition de l'être humain, d'une "pulsion psychique » en termes choisis par von Essen (1967, p. 274), à identifier toutes les sensations par rapport aux impressions perceptives stockées antérieurement dans le cerveau. Sur le plan phonético-phonologique, il s'agit alors des habitudes perceptives et articulatoires transférées de la L1 vers la langue cible (L2). En l'occurrence, pour la 
voyelle /y/, les sons les plus comparables au niveau articulatoire, voisins sur l'axe antérieur/postérieur du système vocalique polonais, seraient le/i/ et le /u/. Comme Guberina (1971, 2003) et Renard (1974) l'ont confirmé dans le cadre de l'enseignement de langues étrangères, celui qui apprend une nouvelle langue perçoit d'une manière erronée, par rapport à un natif, de nouveaux sons et mélodies dans la mesure où le cerveau, n'étant pas encore habitué, n'est pas capable d'interpréter correctement le signal sonore inconnu. En effet, ce dernier se trouve alors dans une zone perceptuelle préexistante (Lhote, 1995) tant que les activations cérébrales de l'apprenant ne seront pas convenablement conditionnées. Comme le souligne Renard (1974) :

Tout système linguistique résulte de l'organisation de données selon un nombre relativement réduit d'unités phoniques nécessaires à la communication. Ce nombre varie d'une langue à l'autre. La distribution phonologique des sons d'une langue donnée va donc conditionner, déterminer le sens discriminatoire de ceux qui l'utilisent. Ainsi, s'établira très tôt, - conséquence logique du conditionnement par approximations successives - une sorte de soumission aveugle (pour ne pas dire sourde) au système phonologique. L'individu demande à sa langue un système de référence, auquel il s'adapte et se soumet. (p. 20)

3 Les similitudes et les différences entre les structures des deux langues (L1 et L2) entrainent automatiquement des transferts positifs facilitant l'apprentissage ou/et des transferts négatifs provoquant certaines difficultés à dépasser (Lado, 1957); cela étant, ce qui est valable pour les systèmes grammatical et lexical, l'est également pour les combinaisons phonétique et phonologique. Apprendre une nouvelle langue, comprendre et s'exprimer oralement signifierait également acquérir de nouvelles habitudes perceptives et articulatoires (von Essen, 1967), éviter la contagion phonétique et phonologique (Mackey, 1976), pouvoir se repérer de manière efficace dans un paysage sonore nouveau (Lhote, 1990). Les travaux de Kuhl (1991), Best (1994) et Flege (1995) ne confirment pas l'hypothèse d'une période critique, sensible, au-delà de laquelle il serait impossible d'acquérir une prononciation quasi authentique qui pourrait faire passer l'apprenant pour un natif. Les habitudes sélectives permettant d'organiser et d'acquérir le système phonologique et les patrons accentuels de la L1 affectent la capacité à percevoir de nouveaux sons, mais les réalisations phonétiques s'approchant toutefois d'un prototype (cas de faux-amis) seraient plus difficiles à discriminer que des exemplaires moins typiques, plus facilement catégorisables perceptivement (Kuhl, 1991).

Dans le cadre de cette étude, nous souhaiterions nous focaliser sur les difficultés de prononciation de la voyelle /y/ par des locuteurs polonophones en FLE de l'université d'Opole (Pologne). Pour faire ressortir ces difficultés, nous avons mené une étude longitudinale sur six locuteurs et les avons accompagnés sur trois étapes consécutives de médiation $\mathrm{du}$ son $/ \mathrm{y} /$ absent dans le système vocalique polonais. Ces trois étapes consécutives correspondent aux trois étapes de recueil de données. Notre objectif est de procéder à une correction phonétique par la méthode verbo-tonale d'intégration phonétique (MVT) pour : en premier lieu, se demander si un cadre de production optimal fondé sur l'intonation et les contextes facilitants permet une prononciation optimale de la voyelle à l'étude; en second lieu, observer de quelle manière peut s'effectuer le réinvestissement des acquis, c'est-à-dire la phase de transfert. Notre analyse contrastive des systèmes contemporains phonologique et phonétique des langues source et cible, corroborée par des analyses acoustiques (Hamm \& Dańko, 2017), avait déjà permis de cerner les pierres d'achoppement lors de l'apprentissage du système phonique et de la production de l'oral en FLE afin d'y apporter une réponse didactique adaptée. Les erreurs 
ne doivent pas se fossiliser, et ce afin de garantir le plus tôt possible une prononciation respectant le français de référence, norme actuellement en vigueur.

\section{Mise en contraste du système vocalique du polonais et du français et les hypothèses qui en découlent}

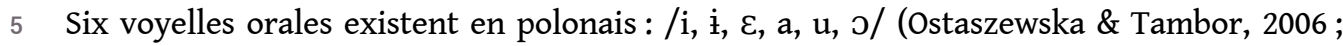
Maciołek \& Tambor, 2012) et ce contre dix voyelles orales de base en français : /i, e, $\varepsilon$, a, y, ø, œ, u, o, J/ (Wioland, 2005). Toutes les voyelles du polonais sont présentes dans le système vocalique français, à l'exception de la voyelle /i/ (fermée sur l'axe ouvert-fermé et médiane sur l'axe antérieur-postérieur d'après Dłuska, 1950/1983). Ce système vocalique est réduit par rapport à celui du français. Les systèmes phonologiques du

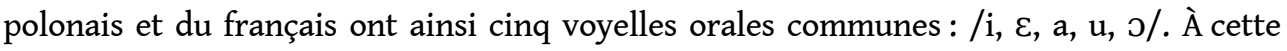
heure, l'état de l'art scientifique sur le traitement de la prononciation des polonophones en FLE est daté et donc, hormis les recherches de Billerey (2004), Gajos (2010) et les nôtres en cours, il est difficile d'obtenir des fondements théoriques et/ou pratiques récents.

6 Notre étude acoustique qui consistait à identifier les caractéristique de «l'accent étranger » de locuteurs polonophones en FLE (Hamm \& Dańko, 2017) nous a permis de confirmer nos hypothèses de départ concernant les interférences à dépasser par tout apprenant polonophone lors de l'apprentissage de l'oral en FLE : les phonèmes qui sont les plus problématiques sont en effet les voyelles orales de moyenne aperture mi-fermées, /e, $\varnothing$, o/ et les deux voyelles antérieures labialisées/y/ et/œ/ du français, car inexistantes dans le système phonologique du polonais. Afin d'observer la structuration des deux systèmes vocaliques, nous avons opté pour une visualisation triangulaire (F1F2). Les travaux récents dans le domaine montrent qu'il faut au moins aller jusqu'à F3 pour une analyse acoustique des voyelles (voire F4 pour le/i/). La limite de ce travail reste la prise en compte des deux premiers formants uniquement, et ce en raison des conditions d'enregistrement réalisés en salle de cours et non plus en salle insonorisée, comme par le passé, rendant la prise de mesures formantiques d'un F3 parfois malaisée. Nous avons ainsi établi les champs de dispersion des voyelles de nos quatre locuteurs dans les deux langues (valeurs brutes et moyennes) et superposé les triangles des moyennes, que nous avons comparés entre eux afin de déterminer le traitement des voyelles de la langue source et de la langue cible dans cet espace commun, mais que nous avons comparés également avec les valeurs de référence de la littérature (valeurs canoniques, fig. 1). Des études récentes sur les valeurs moyennes des formants vocaliques du français ont été menées par Georgeton et coll. (2012) pour les voyelles isolées (calculées à partir de 1600 voyelles isolées : 10 voyelles $\mathrm{x} 4$ répétitions $\mathrm{x} 40$ locutrices). Nous avons utilisé ces dernières pour établir le triangle vocalique du français en vert (valeurs formantiques extrêmes, créant ainsi un triangle vocalique maximal) et l'avons superposé au triangle vocalique du polonais en rouge d'après Wierzchowska (1980) (étude également réalisée sur des voyelles isolées). 
Figure 1. - Superposition des espaces vocaliques du polonais et du français, moyennes $(\mathrm{Hz})$.

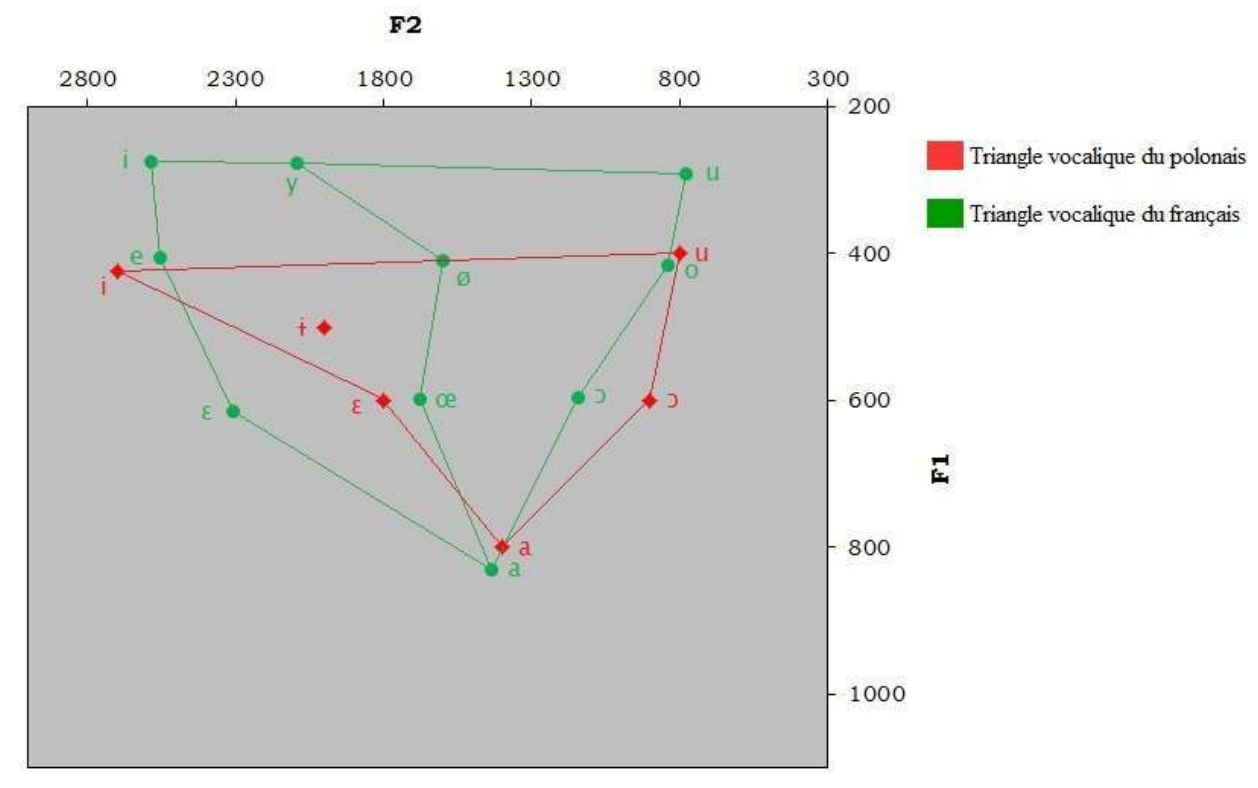

7 Les données de chaque langue sont issues d'études différentes, réalisées à des époques différentes, et les éléments que nous avançons par rapport à cette superposition méritent d'être nuancés mais, dans l'étude de Hamm et Dańko (2017), nous avons démontré que les locuteurs prononçaient soit un /y/ trop postérieur et donc proche de /u/, soit un /y/ trop antérieur et donc proche de /i/. Parfois, le /u/, pourtant commun, était affecté en retour et s'antériorisait se confondant alors avec la production d'un $/ y /$.

Notre étude perceptive (Dańko, Sauvage \& Hirsch, 2015) a, quant à elle, mis en évidence que tous les groupes d'apprenants polonophones (aussi bien de niveau débutant que de niveaux avancés) sont capables de percevoir le son $/ y /$, au moins en contexte très facilitant dans la perspective MVT. Les tests par paires minimales semblaient moins efficaces que les énoncés structurés en groupes rythmiques. Concernant les groupes des niveaux B2-C1 (en cours d'acquisition) et B1-B2 (en cours d'acquisition), les difficultés concernaient les énoncés plus longs (10 syllabes), les pseudo-énoncés (quand le sens ne pouvait jouer son rôle dans l'aide à la perception) et en contextes défavorables (en position médiane, non accentuée, dans un entourage consonantique défavorable avec une intonation descendante). Concernant le groupe de niveau A2-B1 (en cours d'acquisition), en plus des difficultés pointées ci-dessus, les apprenants peinaient également à discriminer la voyelle /y/ au début des groupes rythmiques. Enfin, les apprenants des niveaux A1 et A1-A2 (en cours d'acquisition) rencontraient en plus des difficultés au niveau des tests par oppositions phonologiques.

9 Nous souhaitons, à présent, agir sur la perception et la production de nouveaux locuteurs grâce aux procédés de la MVT et les accompagner dans leur entrainement (répétitions de stimuli/lecture, production orale guidée et semi-spontanée en FLE), objets de notre étude longitudinale, afin de permettre l'acquisition/perfectionnement du son/y/ puisqu'il s'agit d'une nouvelle catégorie phonologique à (re)construire. Pour ce faire, nous décrivons ci-dessous les résultats de cette expérimentation qui a été menée à l'université 
d'Opole : il s'agit d'une étude sur trois phases et comprenant des éléments d'analyse acoustique précisés dans le protocole expérimental suivant.

\section{Protocole expérimental}

\subsection{La méthodologie et les corpus}

Nous avons choisi d'utiliser la MVT (Intravaia, 2000 ; Guberina, 2003) et de proposer un travail novateur dans le sens où les études longitudinales menées par ce moyen de correction phonétique et présentant véritablement des résultats scientifiques sont rares. Cette méthode est un savoir-être et surtout un savoir-faire artisanal, une démarche interventionniste qui procède par nuancements et distorsions sur tous les paramètres du langage. Le diagnostic est posé selon l'axe de la tension (physiologie) ou de l'acuité (perception) et les procédés peuvent s'employer seuls mais surtout s'enchainer, se combiner entre eux (« cumul des procédés », Intravaia, 2000). En effet, divers procédés comme la prononciation déformée ou nuancée et les « entourages facilitants 》 (procédés d'appoint) peuvent être utilisés isolément, mais sont bien plus efficients travaillés à l'intérieur d'un mouvement rythmico-intonatif, véritable charpente, que la gestualité (macro-motricité) viendrait accompagner. Le but de cette méthode est de faire entendre un phonème de manière optimale afin de rendre l'erreur physiquement impossible, l'apprenant procédant alors par approximations successives sous le contrôle de l'audition. Traiter spécifiquement la perception, en optimiser les conditions au sein d'un énoncé qui se suffit à lui-même, peut s'avérer constituer une aide précieuse : l'apprenant sera doté d'une compétence stratégique passant par l'audiophonatoire et son oreille "dégagée » afin de permettre l'accès à toutes les autres compétences (communicative, lexicale, morpho-syntaxique, discursive...). L'approche est de plus personnalisée puisqu'il s'agit de fournir le procédé idoine à tel ou tel apprenant et en temps réel.

11 Notre protocole expérimental comprend trois phases d'obtention des données. La première étape (I) fut diagnostique : elle a consisté à réaliser un enregistrement de nos locuteurs lors de la lecture d'un petit dialogue (fig. 2, support $\mathrm{n}^{\circ} 1$ ci-dessous; corpus contrôlé, oralisé, tiré de Fauré \& Di Cristo, 1977, p. 22) et d'une narration préparée en 30 minutes et induite par des mots-clés (fig. 3 , support $n^{\circ} 2$ ci-dessous) dont l'apprenant avait pris connaissance grâce à des étiquettes ou à des images. Le petit dialogue a été choisi en raison de son extrême richesse en occurrences de voyelles de petite aperture. La thématique de la narration était celle des vacances et de la visite d'une ville. Les mots-clés étaient généralement connus des apprenants, car figurant dans leur manuel de langue. La consigne était la suivante : «Parlez de vous, de vos habitudes et préférences en utilisant les éléments lexicaux indiqués (préparation : 30 minutes). » Les voyelles à l'étude étaient placées en fin de mot, donc accentuables, ce dans l'espoir d'en obtenir un maximum accentuées en production puisque c'est en fin de mot phonétique que la réalisation d'un phonème est optimale, l'accent du français étant duratif. Cette fin de mot phonétique est aussi garante d'une bonne perception/compréhension des messages (Callamand, 1981; Lhote, 1990; Wioland, 2005). Les données ainsi recueillies nous ont permis de mener une analyse acoustique distinguant les aires de dispersion des voyelles $/ \mathrm{i}, \mathrm{y}, \mathrm{u} /$ propres à chaque locuteur (au moins 20 occurrences attendues de chaque voyelle, soit 60 occurrences pour chaque locuteur et au total 360 voyelles). 
Figure 2. - Support $\mathrm{n}^{\circ} 1$ (Phase I).

$-D^{\prime}$ 'où venez-vous ?

-Nous venons du cours de littérature russe.

-Ça vous a plus?

-Beaucoup! Mais c'est un cours difficile!

-Difficile? Mais vous êtes stupide ! J'ai toujours entendu dire que ce cours était très facile.

-C'est vous qui le dites.

12 Nous avons ainsi 6 occurrences de /i/ : difficile, difficile, stupide, dire, facile, dites ; 4 de / $\mathrm{y} /$ : littérature, russe, plu, entendu ; et $9 \mathrm{de} / \mathrm{u} /:$ d'où, vous, tous, cours, beaucoup, cours, toujours, cours, vous.

Figure 3. - Support $\mathrm{n}^{\circ} 2$ (Phase I).
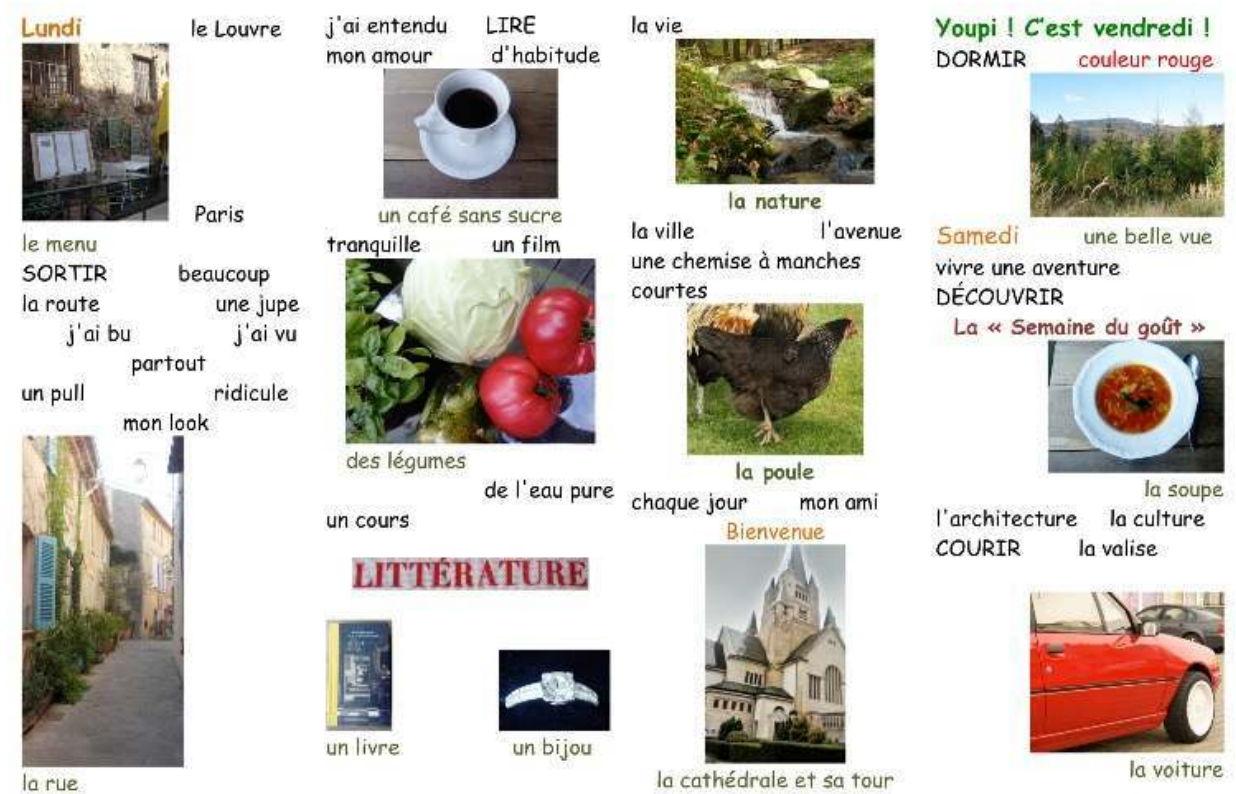

la culture COURIR

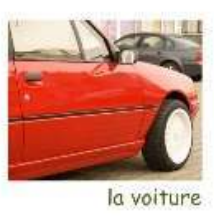

Le reste des occurrences :

- /i/ : tranquille, Paris, dormir, la vie, un film, mon ami, sortir, lire, courir, découvrir, lundi, samedi, Youpi ! C'est vendredi !, la ville, une chemise, la valise; les images: un livre (18 occurrences) - au total 24 occurrences sur les supports $n^{\text {os }} 1$ et $2(6+18)$;

- /y/ : j'ai bu, j'ai vu, j'ai entendu, vivre une aventure, de l'eau pure, ridicule, l'architecture, la culture, d'habitude, l'avenue, bienvenue, une jupe, un pull; les images : des légumes, un café sans sucre, la rue, la littérature, la nature, une belle vue, la voiture, le menu (21 occurrences) - au total 25 occurrences sur les supports $n^{\text {os }} 1$ et $2(4+21)$;

- /u/ : la route, mon look, une chemise à manches courtes, un cours, mon amour, beaucoup, partout, chaque jour, le Louvre, la "Semaine du goût », couleur rouge ; les images : la poule, la soupe, la cathédrale et sa tour, un bijou (15 occurrences) - au total 24 occurrences sur les supports $n^{\text {os }} 1$ et $2(9+15)$.

Pour tous nos corpus, nous avons permis aux locuteurs de ne pas respecter scrupuleusement les déterminants indiqués : ils pouvaient effectuer une substitution s'ils le désiraient. 
15 Notre hypothèse concernant cette étude, formulée en tenant compte des interférences prévues, était que certains étudiants auront tendance à surestimer les composantes sombres de la voyelle /y/ (nous devrions alors observer des valeurs acoustiques tendant vers $/ \mathrm{u} /$ ) et que d'autres auront tendance à surestimer les composantes claires de cette voyelle (nous devrions alors découvrir des valeurs acoustiques tendant vers $/ \mathrm{i} /$ ).

16 Une première évaluation perceptive des propriétés subjectives des productions $\mathrm{du} / \mathrm{y} /$ avait été menée en amont par l'enseignant en classe de langue, et cette observation informelle a ainsi été corroborée par une visualisation triangulaire F1/F2, donc également interprétable en termes articulatoires, présentant la tendance de chaque locuteur afin de leur fournir le corpus de remédiation idoine.

17 La deuxième étape (II) a consisté à proposer une remédiation, en fonction des résultats précédemment obtenus et en réponse aux difficultés réellement rencontrées par nos locuteurs. Selon les procédés d'appui ou d'appoint préconisés par la MVT (Callamand, 1981 ; Guimbretière, 1996 ; Intravaia, 2000; Billières, 2005), nous avons construit deux corpus de phrases porteuses de la voyelle /y/, le plus souvent composées d'un ou de deux mots phonétiques maximum (au moins 25 occurrences par corpus); la voyelle /y/ était placée dans un contexte favorable à la correction du son (trop clair ou trop sombre). Nous avons privilégié l'intonation (une intonation montante met en valeur les fréquences claires d'un /y/ accentué placé en fin de courbe intonative et une intonation descendante mettra en valeur les composantes sombres de ce même/y/) et aussi l'emploi d'un contexte facilitant. Les voyelles éclaircissantes sont /s, z, t, d, n/, les assombrissantes /p, $\mathrm{b}, \mathrm{m}, \mathrm{f}, \mathrm{v} /$. De même, un débit rapide aurait tendance à tendre les voyelles et un rythme plus lent à les détendre. La tâche de chaque étudiant a consisté à écouter et à répéter les énoncés du corpus (stimuli) qui lui était dédié en fonction de ses difficultés (voir le tableau ci-dessous, fig. 4).

Figure 4. - Phase II.

\begin{tabular}{|l|l|}
\hline Remédiation 1 & Remédiation 2 \\
\hline $\begin{array}{l}\text { Quand le /y/tend vers le /u/ } \\
\text { (surestimation des composantes sombres) }\end{array}$ & $\begin{array}{l}\text { Quand le /y/ tend vers le /i/ } \\
\text { (surestimation des composantes claires) }\end{array}$ \\
\hline $\begin{array}{l}\text { Contexte facilitant: position accentuée, fin de } \\
\text { courbe rythmique ascendante, entourage des } \\
\text { consonnes éclaircissantes /s, z, t, d, n/, } \\
\text { rythme rapide }\end{array}$ & $\begin{array}{l}\text { Contexte facilitant : position accentuée, fin de } \\
\text { courbe rythmique descendante, entourage des } \\
\text { consonnes assombrissantes /p, b, m, f, v/, } \\
\text { rythme lent }\end{array}$ \\
\hline
\end{tabular}




\begin{tabular}{|c|c|}
\hline Quelle aventure? & Pardon, je n'ai pas vu. \\
\hline Il fait des études? & C'est vraiment touffu. \\
\hline En train ou en voiture? & Désolée, je n'ai pas pu. \\
\hline La Côte d'Azur? & Non, je n'ai pas bu. \\
\hline Ils n'ont pas répondu? & Ce n'est qu'un début. \\
\hline Il est sûr? & Il est malade et il fume. \\
\hline Il a mal à la nuque? & D’accord, c'est prévu. \\
\hline C'est si dur? & La tarte aux mûres. \\
\hline Cinq minutes? & Un employé promu. \\
\hline Quel tube? & Avez-vous vu... \\
\hline Il est sûr de sa pointure? & Un murmure, comme une plume. \\
\hline C'est une ridicule pustule. & C'est une armure. \\
\hline Ses cheveux sont hirsutes? & Mais non, l'eau est pure. \\
\hline Turlututu, le chapeau pointu! & Non, je n'aime pas cette coiffure... \\
\hline Tu l'as su? & C'est un bon jus. \\
\hline Vers le Sud? & Un efficace vermifuge. \\
\hline Une Suze? Vous voulez une Suze? & Quelle bûche... \\
\hline Encore aurait-il fallu qu'ils le sussent! & Une robe de bure. \\
\hline Tu n'as pas de ceinture? & La saison de mue. \\
\hline Je suis archi déçu de cette puce! & Nous le bûmes. \\
\hline Tu dis? Il se situe? & On est émus. \\
\hline Tu y chutes? & On est rompus. \\
\hline Ici : issue. & Le bon tofu. \\
\hline Ils en usent? & Un homme barbu. \\
\hline Flûte ! Zut, zut et zut! & \\
\hline Message reçu. & \\
\hline
\end{tabular}

L'objectif principal a été de confronter les données ainsi recueillies (étapes I et II) afin de comparer les résultats : nous espérions pouvoir observer l'aire de dispersion $\mathrm{du} / \mathrm{y} / \mathrm{se}$ déplacer très manifestement lors de la phase de répétition de ces stimuli, afin de prouver qu'un contexte de perception optimal, prédéfini en fonction des besoins concrets de nos locuteurs, permet la médiation du son $/ \mathrm{y} /$.

19 Nous avons in fine terminé notre étude par l'enregistrement et l'étude perceptive et acoustique d'une seconde narration (étape III) à l'issue d'une courte période de remédiation par la MVT (cours de phonétique à l'université d'Opole durant un mois à raison de $1 \mathrm{~h} 30 /$ semaine +10 minutes de travail individuel à la maison chaque jour). Pour cette phase III, nous avons redonné des étiquettes et des images à nos locuteurs, ce afin d'obtenir à nouveau au moins 20 occurrences de la voyelle /y/. Ils avaient 20 minutes de préparation pour construire un récit cohérent.

20 Voici les mots-étiquettes donnés : d'habitude, j'ai obtenu, l'architecture, l'avenue, la rue, j'ai reçu, la lecture, j'ai bu, j'ai vu, j'ai entendu, j'ai lu, j'ai su, j'ai vécu une aventure, j'ai connu, c'est nul, culture, c'est ridicule, c'est sûr, c'est de plus en plus dur, j'ai eu, j'ai vend $\mathbf{u}$; les images : une belle vue, la nature, ma voiture (24 éléments). 
Figure 5. - Support $\mathrm{n}^{\circ} 3$ (Phase III /y/).

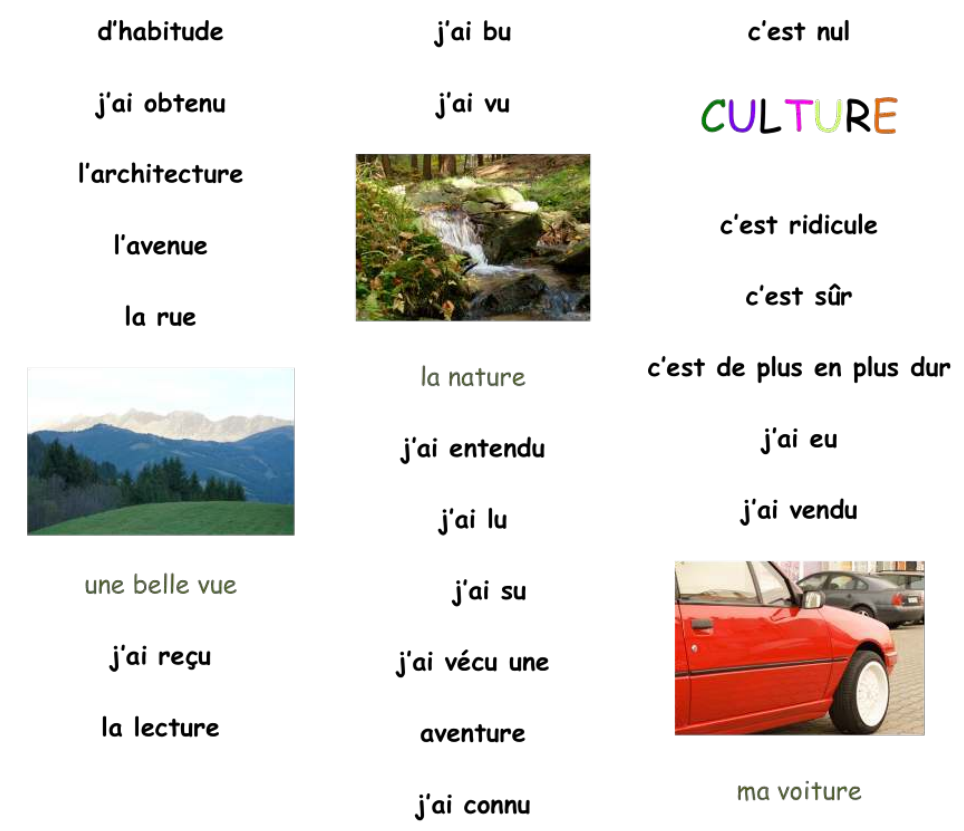

21 Deux locuteurs sur six présentaient un $/ \mathrm{u} /$ affecté en retour. Il s'agit d'un phénomène que nous avons déjà observé dans notre étude de 2017 (Hamm \& Dańko). L'apprenant se focalisant sur la prononciation $\mathrm{du} / \mathrm{y} /$, d'une manière incontrôlée, prononce certains $/ \mathrm{u} /$ de manière trop antériorisée, pouvant être ainsi confondu avec un $/ y /$. Pourtant, la voyelle $/ \mathrm{u} /$ existe dans le système phonologique polonais, elle est proche $\mathrm{du} / \mathrm{u} /$ français au niveau articulatoire sur l'axe antérieur/postérieur. La prononciation d'un $/ \mathrm{u} /$ ne pose généralement aucun problème, sauf dans le cadre d'un entrainement sur le /y/, et ne concerne que certains apprenants; elle revient pourtant de façon chronique dans les groupes d'apprenants se succédant au fil du temps.

22 Nous avons donc décidé, pour la phase III, de faire travailler nos deux locuteurs, concernés par ce phénomène, à la fois sur le/y/ et sur le /u/. Les deux étudiants disposaient de 30 minutes de préparation.

Les indices lexicaux étaient les suivants : j'ai pris la route, amour, chaque jour, beaucoup, partout, pas du tout, sans doute, à Tours, à Toulouse, dans la cour, c'était fou, c'était cou rt, le restaurant «Sur la route du retour », moules frites, cuisses de grenouilles, poulet au chou rouge, légumes du jour, courgettes au four, coupe de fruits rouges, prunes cuites au sucre roux ; les images : la roue de secours, la cathédrale et sa tour, une poule, une mou che (24 éléments). 
Figure 6. - Support $\mathrm{n}^{\circ} 4$ (Phase III /u/).

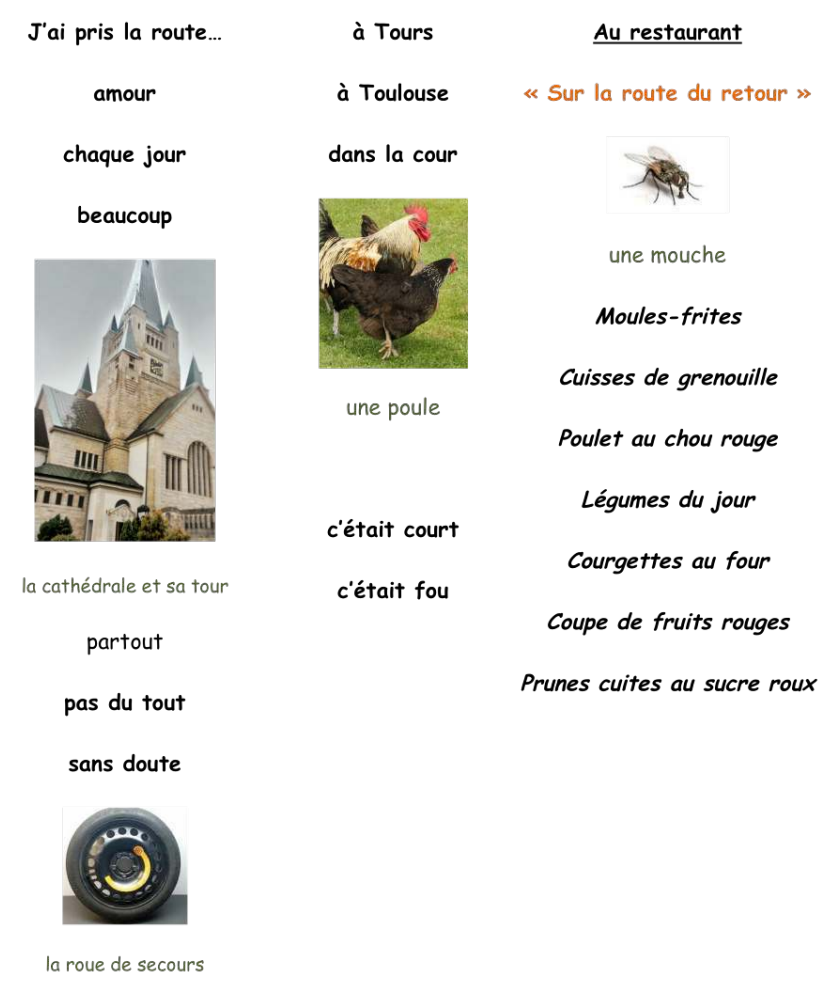

\subsection{Les locuteurs}

Six étudiants (trois femmes et trois hommes) appartenant à l'université d'Opole ont participé à cette étude : deux de niveau A1 et 4 de niveau A2. Nos pratiques de classe nous avaient déjà permis d'établir que les locuteurs 1 et 2 avaient tendance à produire un $/ \mathrm{y} /$ trop sombre, les locuteurs 3 et 4 , quant à eux, produisaient un $/ y /$ trop clair. Les locuteurs 5 et 6 présentaient un $/ \mathrm{u} /$ affecté en retour, prononcé trop clair pour certaines de ses occurrences. Nous les avons regroupés par catégories en fonction du résultat de l'étape diagnostique qui est venue confirmer nos premières observations perceptives.

\subsection{Les enregistrements et les mesures}

Les enregistrements ont été effectués au laboratoire de langues de l'université d'Opole équipé de postes individuels avec ordinateurs (références: UO-IFP-NC-223 Workgroup, G Factor Digital, Windows 10Pro 160714393.2007 Intel $^{\circledR}$ Core (TM) i3-3240CPU 3,40 GHz 4,00 GB) : le 27 avril 2017 (phase I), le 11 mai 2017 (phase II), le 9 juin 2017 (phase III). Le son a ensuite été transféré vers un ordinateur de l'Institut de phonétique de Strasbourg où tous les signaux acoustiques (formants et continuum sonore) apparaissent à l'écran grâce au logiciel d'analyse acoustique Praat (Boersma \& Weenink, 2010). Nous avons relevé les valeurs nécessaires à notre analyse, à savoir les deux premiers formants (F1 et F2) responsables du timbre des voyelles concernées par l'étude. Les mesures, toutes manuelles, ont été prises au milieu de la structure formantique stable des voyelles. Nous les avons vérifiées grâce à l'onglet Formant listing. Les mesures ont été reportées sur des 
graphiques et ont servi à élaborer des analyses acoustiques mettant à l'épreuve nos précédentes hypothèses.

\section{Recueil et analyses des données}

Nous avons établi, à partir de l'étude acoustique des différents corpus (trois phases pour rappel), un recueil de données s'organisant de la façon suivante: à chaque locuteur correspondent trois triangles vocaliques, un pour chaque étape. Ils présentent les zones de dispersion des trois voyelles de petite aperture /i, y, u/. Nous avons mis en regard les phases I et II ; la phase III, présentant le réinvestissement des compétences, plus grande, est ainsi mise en avant.

\section{1. Étude formantique des trois étapes, locuteurs 1 et 2}

\subsubsection{Locutrice 1}

Figure 7. - Valeurs formantiques brutes $(\mathrm{Hz})$ : phases I et II.

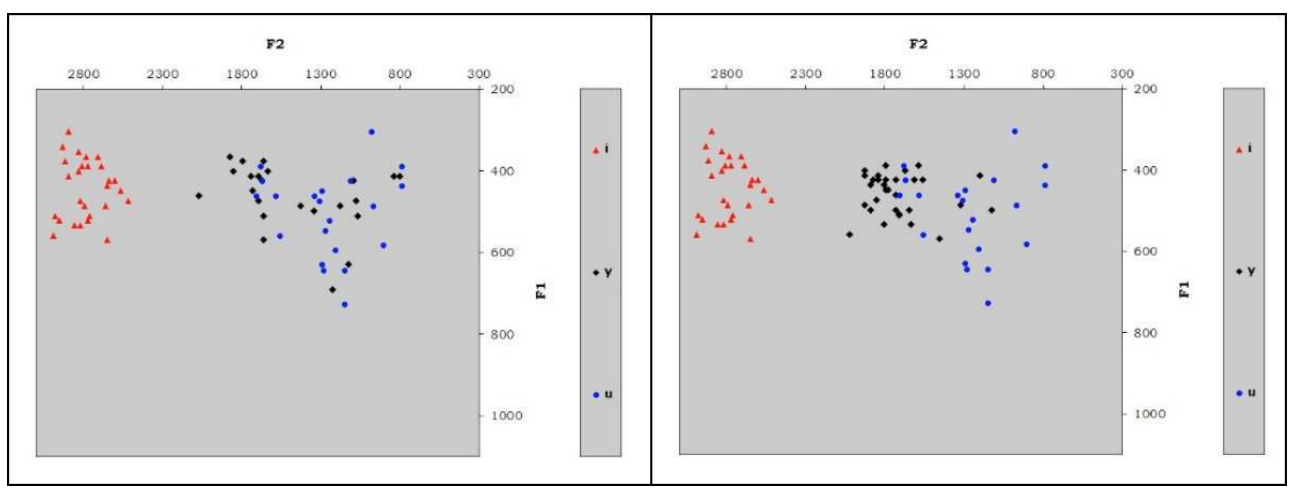


Figure 8. - Valeurs formantiques brutes $(\mathrm{Hz})$ : phase III.

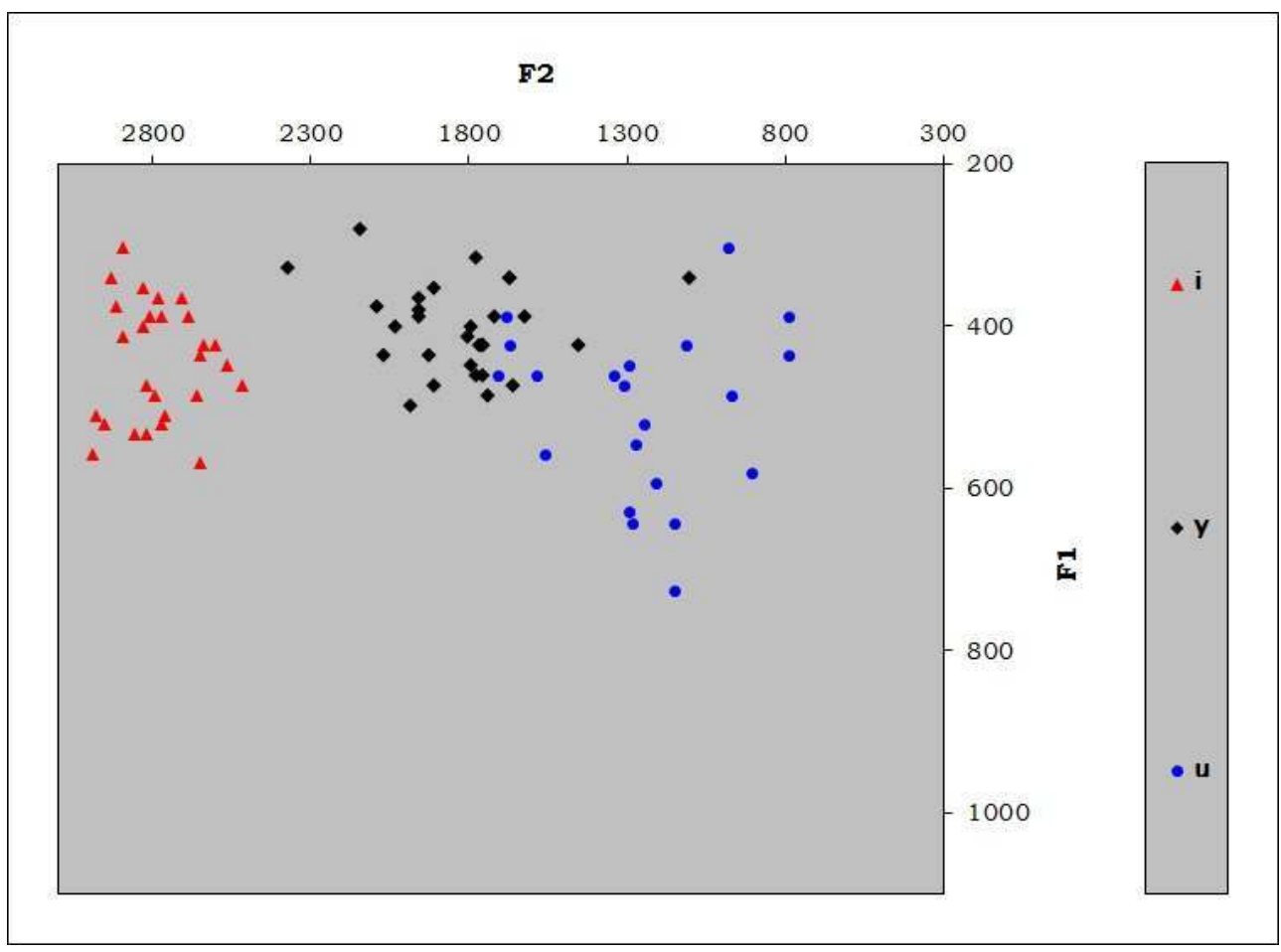

27 En phase I, l'aire de dispersion des valeurs formantiques (F1 et F2) de la voyelle /i/ présente des points regroupés de manière homogène et elle est bien en place dans la représentation triangulaire par rapport à ce qui attendu. Le F1 varie de 304 à $570 \mathrm{~Hz}$. Par contre, on observe clairement une confusion sur l'axe clair/sombre des voyelles /y/ et / $\mathrm{u} /$. Certains / $\mathrm{y} /$ sont trop sombres et empiètent dans la zone $\mathrm{du} / \mathrm{u} /$ et les deux aires de dispersion sont grandes: elles présentent une large étendue sur l'axe duF1, ce qui correspond à un degré d'aperture peut-être mal contrôlé par l'apprenant (entre 304 et $727 \mathrm{~Hz}$ ), alors que le /u/ existe dans le système phonologique du polonais.

En phase II, nous avons décidé de garder en guise de contrôle nos valeurs diagnostiques $\mathrm{de} / \mathrm{i} /$ et de $/ \mathrm{u} /$ (valeurs contrôles permettant l'étude du déplacement de l'aire de dispersion $\mathrm{du} / \mathrm{y} /$ ) et de donner la remédiation $\mathrm{n}^{\circ} 1$ (voir fig. 4) à cette locutrice (éclaircissement de la voyelle/y/). Lors des répétitions, l'aire de/y/ s'est bien antériorisée et différenciée de celle $\mathrm{du} / \mathrm{u}$ / et son étendue sur l'axe de F1 s'est amoindrie : entre 389 et $570 \mathrm{~Hz}$. Quatre occurrences ont été prononcées malgré tout / $\mathrm{u} /$ : « usent », «voiture ", " pointu» et "Côte d'Azur ». L'efficacité de cette phase de répétition de stimuli est très nette.

Lors de la phase d'accompagnement par la méthode MVT in situ, nous avons réussi à faire antérioriser encore plus et surtout à faire tendre la voyelle erronée (entre 280 et $498 \mathrm{~Hz}$ ). C'est une avancée non négligeable, car au cours de notre précédente étude (Hamm \& Dańko, 2017) nous avons pu établir que les Polonais avaient des difficultés à tendre les voyelles fermées françaises. La voyelle /y/ est à présent en place, sauf pour deux occurrences prononcées $/ \mathrm{u} /:$ : bu » et « vu ».

\subsubsection{Locuteur 2}


Figure 9. - Valeurs formantiques brutes $(\mathrm{Hz})$ : phases I et II.

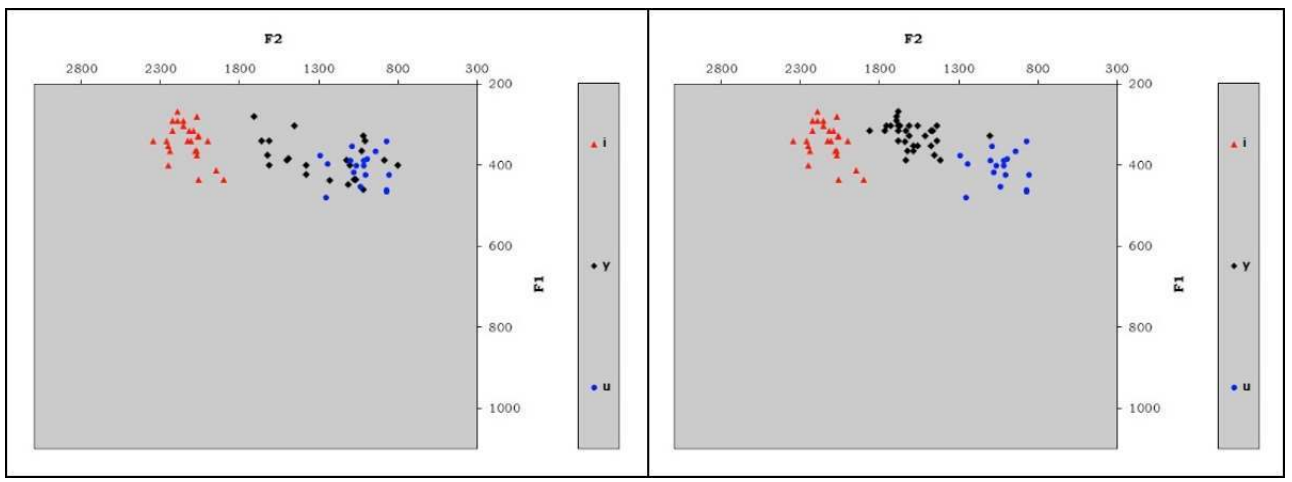

Figure 10. - Valeurs formantiques brutes $(\mathrm{Hz})$ : phase III.

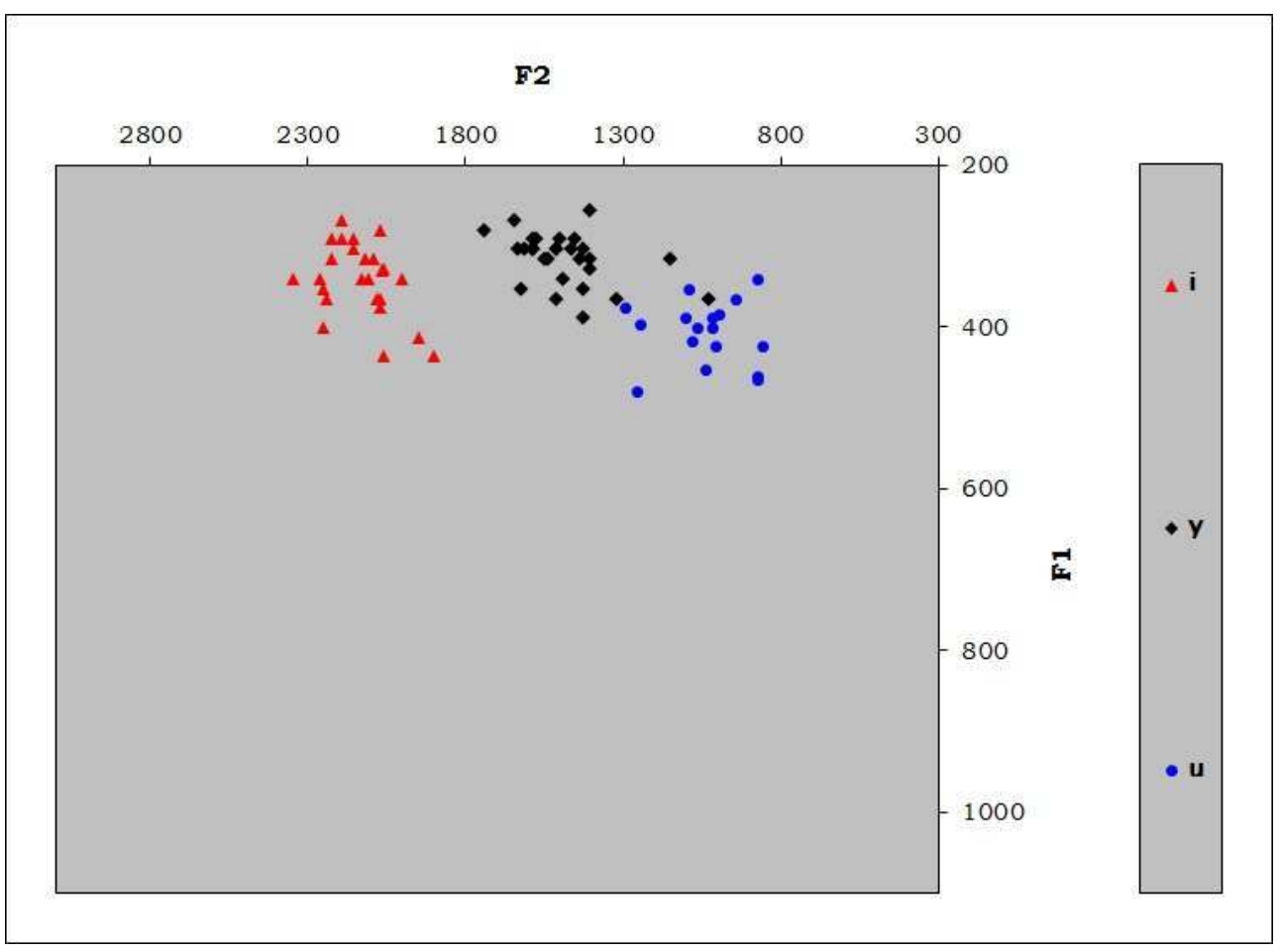

Ce locuteur présente les mêmes erreurs de prononciation en phase I que la locutrice précédente : la moitié des occurrences de la voyelle/y/ se confondent avec l'aire de dispersion $\mathrm{du} / \mathrm{u} /$, qui lui est bien maitrisé. Les valeurs de F1 pour les trois voyelles sont tout de même plus harmonieuses. Nous lui avons donné la remédiation $\mathrm{n}^{\circ} 1$ (voir fig. 4 , éclaircissement).

La phase de répétition des stimuli s'est montrée très convaincante: l'aire parait homogène par rapport aux aires de dispersion canoniques (une seule occurrence est prononcée $/ \mathrm{u} /:$ : «issue ») et nous pouvons constater qu'elle s'est maintenue telle lors de la phase de réinvestissement/transfert des connaissances avec malgré tout une petite tendance vers l'assombrissement, mais les points sont restés globalement groupés (sauf pour deux occurrences « nul » et « obtenu»). 


\section{2. Étude formantique des trois étapes, locuteurs 3 et 4}

\subsubsection{Locuteur 3}

Figure 11. - Valeurs formantiques brutes $(\mathrm{Hz})$ : phases I et II.

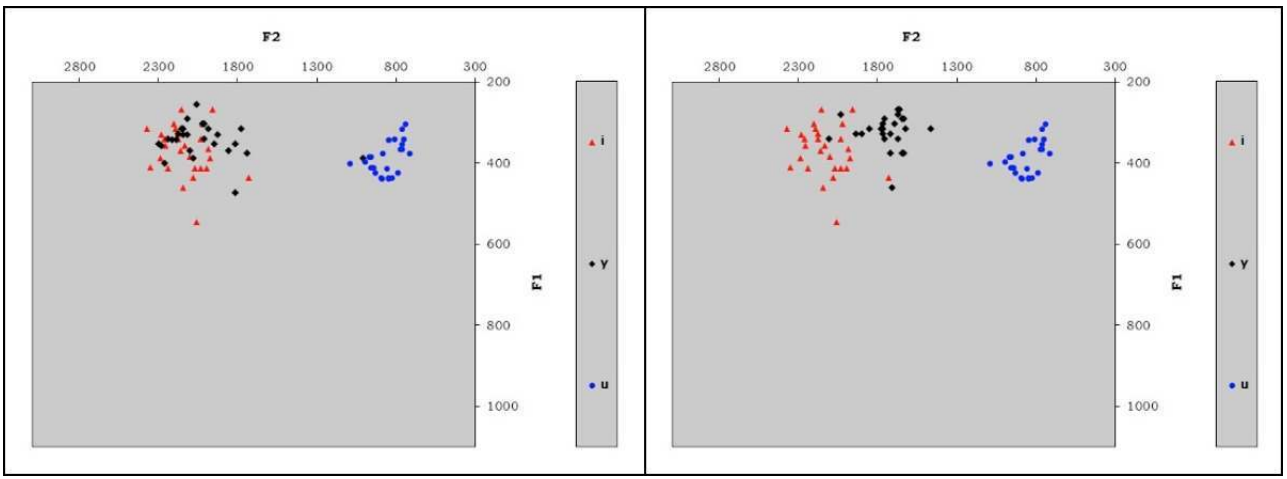

Figure 12. - Valeurs formantiques brutes $(\mathrm{Hz})$ : phase III.

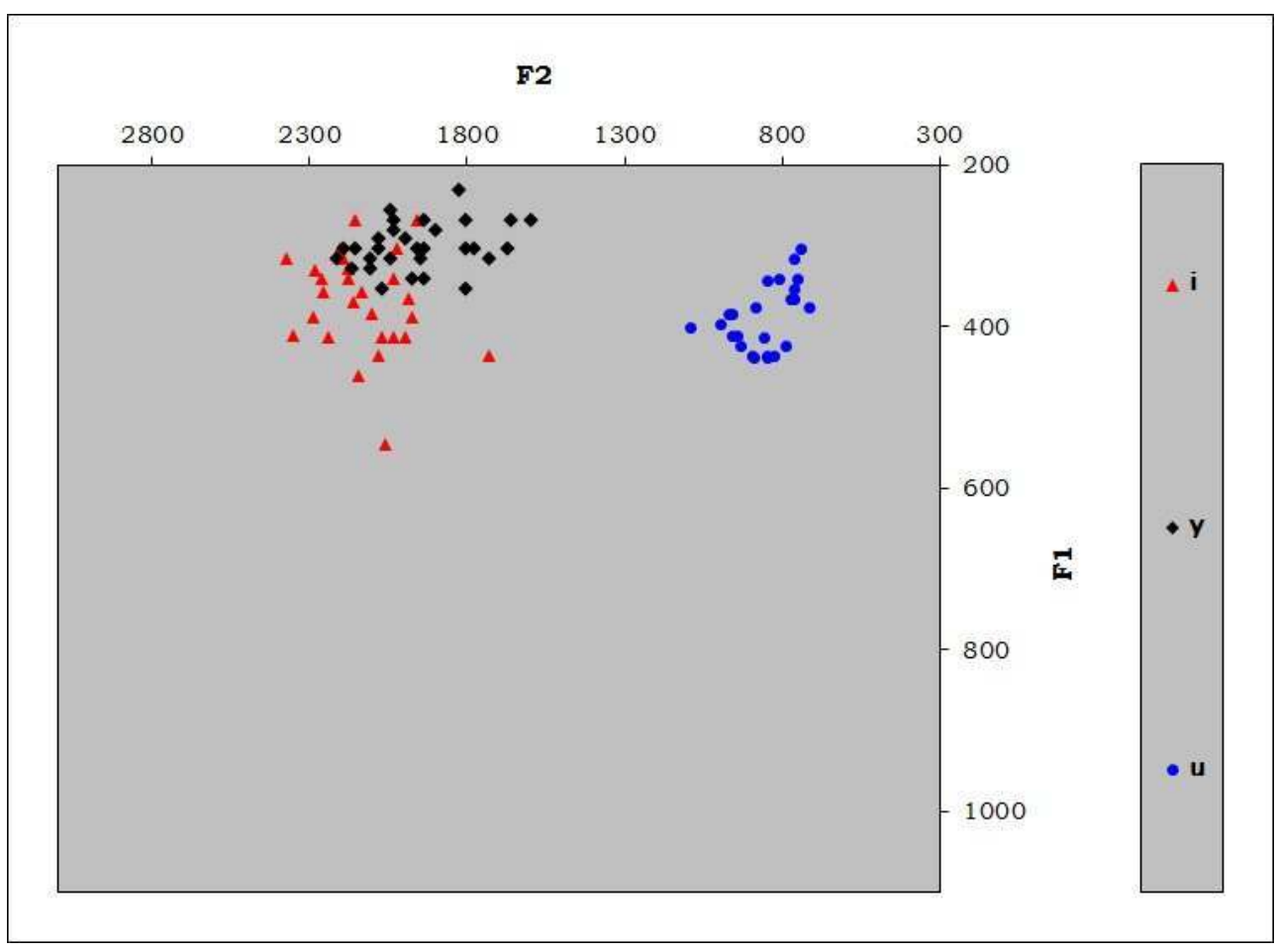

Une nette confusion est visible en phase I entre les aires de dispersion $\mathrm{du} / \mathrm{i} / \mathrm{et} \mathrm{du} / \mathrm{y} /$, ces aires sont homogènes et s'étendent entre 256 et $546 \mathrm{~Hz}$ sur l'axe de $\mathrm{F} 1$. La configuration de l'aire $\mathrm{du} / \mathrm{u} /$ permet par contre de conclure que la production de cette voyelle est maitrisée. Une seule occurrence a été prononcée /u/ dans le mot «jupe ».

Nous lui avons donné la remédiation $\mathrm{n}^{\circ} 2$ (voir fig. 4, assombrissement) et globalement cette phase de remédiation a été efficace, sauf pour deux occurrences «mûres » et « pure » prononcées avec un /i/. 
Le système phonologique de ce locuteur s'est montré particulièrement résistant lors de la phase III, nous observons une régression. Le / $\mathrm{y} /$ est globalement très tendu et plutôt clair, mais son aire de dispersion reste tout de même bien distincte de celle du /i/.

\subsubsection{Locutrice 4}

Figure 13. - Valeurs formantiques brutes $(\mathrm{Hz})$ : phases I et II.

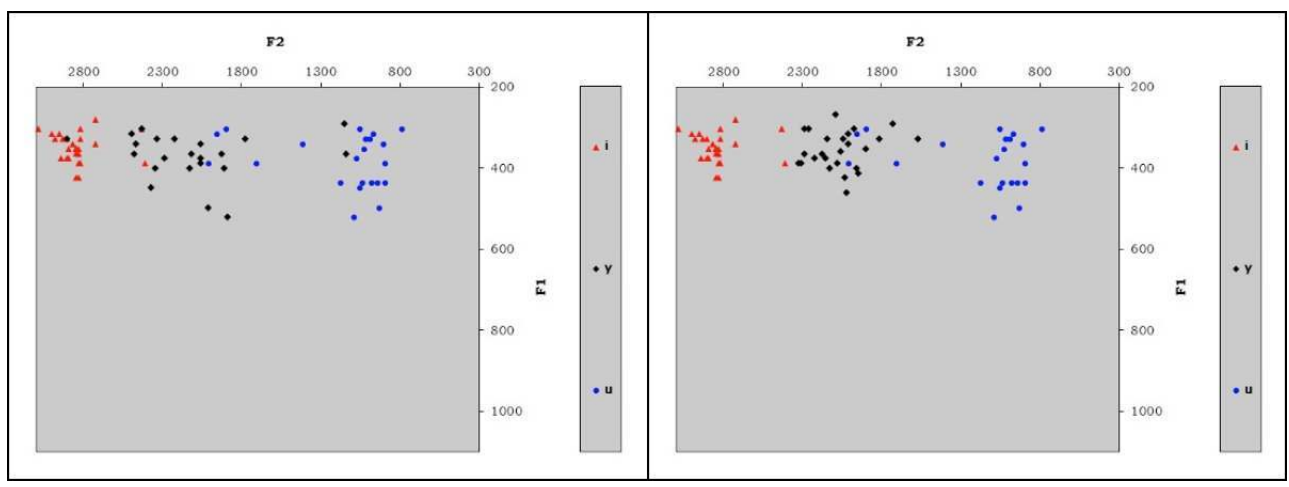

Figure 14. - Valeurs formantiques brutes $(\mathrm{Hz})$ : phase III.

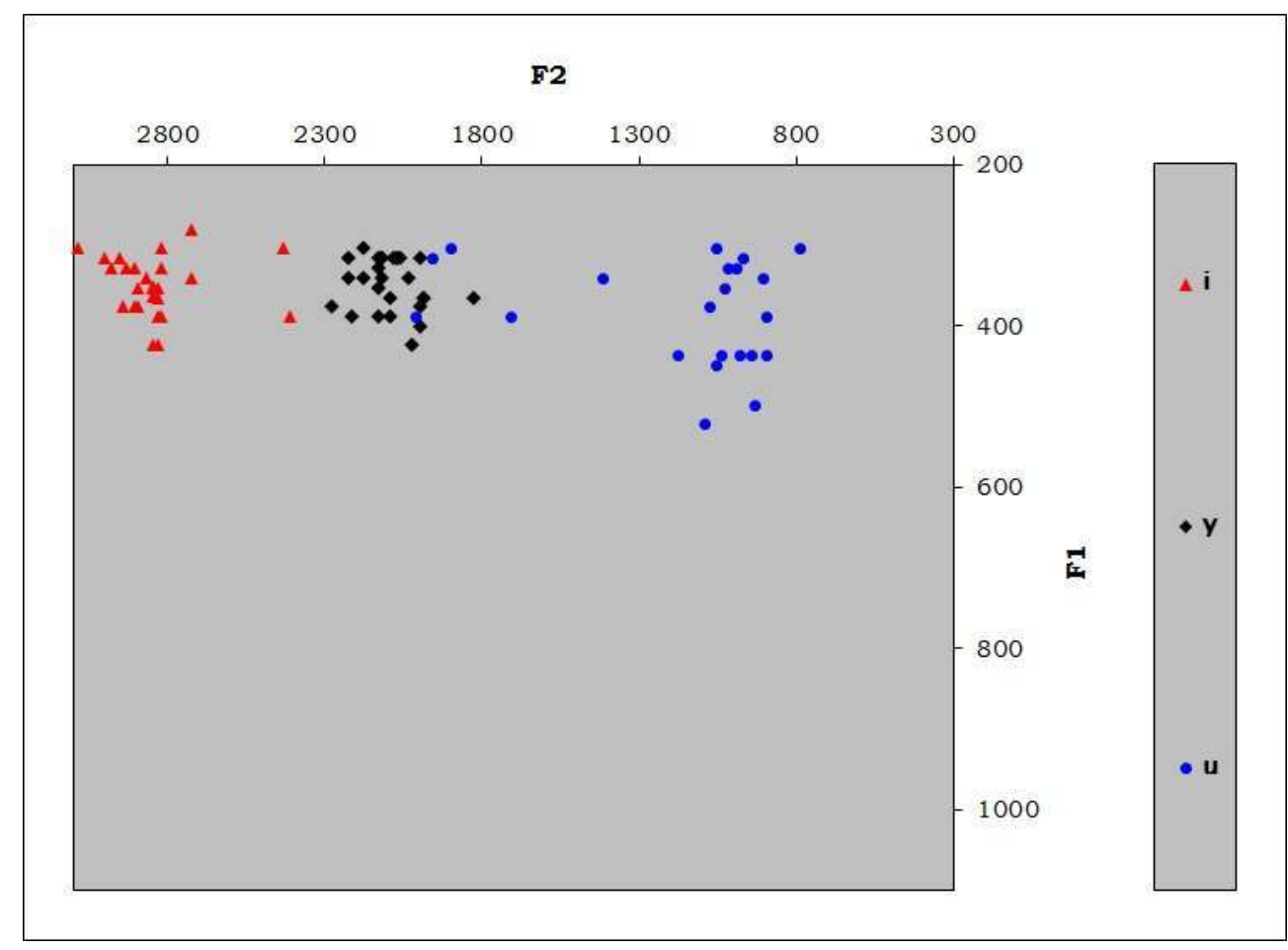

Cette locutrice présente une aire de dispersion des/i/ conforme à nos hypothèses de départ, mais 5 occurrences de/y/ ont été prononcées/i/ (dans les mots "plu», "d'habitude», «légumes", " couru», "vue»), 2 autres occurrences de /y/ ont été prononcées $/ \mathrm{u}$ / (dans les mots « russe» et « architecture ») et 5 occurrences de / $\mathrm{u} /$ ont été prononcées /y/ (dans les mots " goût ", « tous ", « vous ", « d'où », « beaucoup »). Le / $\mathrm{u} /$ semble avoir été légèrement affecté alors que cette voyelle est présente dans le système phonologique polonais. L'étendue large des /y/ et /u/ (entre 292 et $521 \mathrm{~Hz}$ ) nous suggère aussi que leur aperture est mal maitrisée : le /y/ est en cours d'acquisition. Nous 
avons décidé de donner la remédiation $\mathrm{n}^{\circ} 2$ (voir fig. 4 , assombrissement) à cette locutrice, car notre évaluation perceptive diagnostique et celle pratiquée en classe de langue à Opole venaient corroborer cette décision.

Nous voulions tenter d'uniformiser l'aire de dispersion du/y/, la sortir de la zone des /i/ et cela a fonctionné. Le travail en aval a affiné la production $\mathrm{du} / \mathrm{y} /$ dans le sens où il est nettement visible que son aire de dispersion s'est resserrée.

\section{3. Étude formantique des trois étapes, locuteurs 5 et 6}

\subsubsection{Locuteur 5}

Figure 15. - Valeurs formantiques brutes $(\mathrm{Hz})$ : phases I et II.

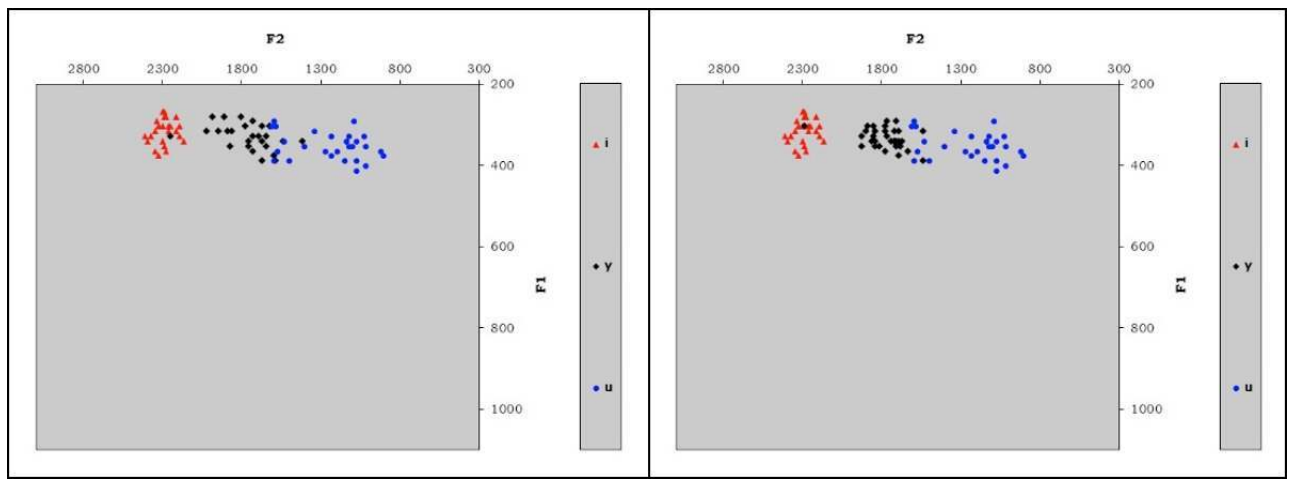

Figure 16. - Valeurs formantiques brutes $(\mathrm{Hz})$ : phase III.

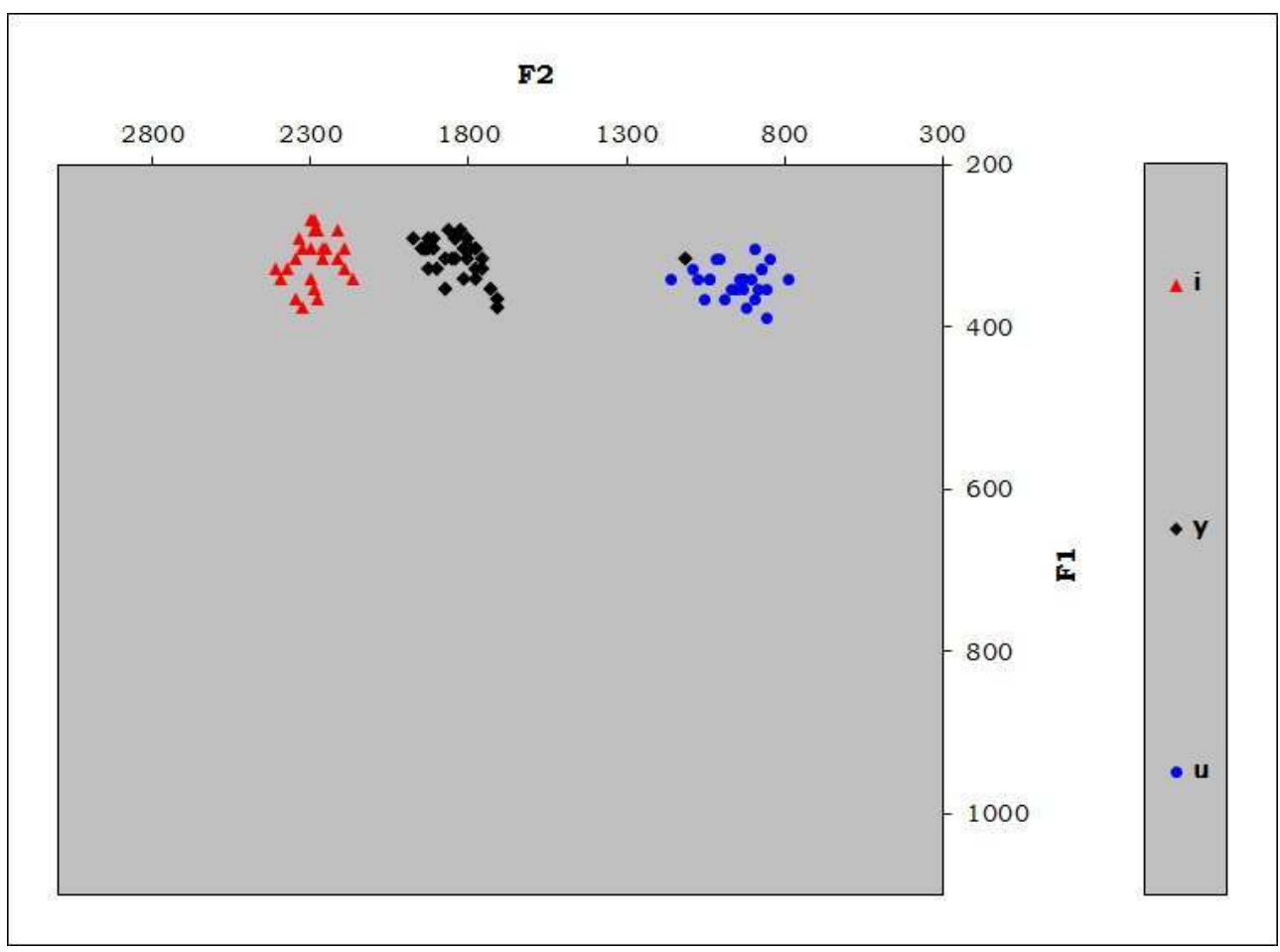

Ce locuteur présente une confusion des $/ y /$ et des $/ \mathrm{u} /$ dans une zone qui s'étend entre 1403 et $1693 \mathrm{~Hz}$, mais l'aperture des trois voyelles fermées est bien contrôlée (268 à $413 \mathrm{~Hz}$ ). Un /y/ a été prononcé /i/: dans le mot «menu ». Ainsi, nous avons décidé de 
faire travailler ce locuteur sur la remédiation $\mathrm{n}^{\circ} 1$ (voir fig. 4, éclaircissement) afin de séparer les $/ \mathrm{y} /$ et $/ \mathrm{u} /$ et de le faire travailler en phase III également sur le $/ \mathrm{u} /$.

En phase II, l'aire du /y/ s'est bien resserrée et la configuration formantique à l'issue de la phase III est correcte, hormis un seul /y/ prononcé / $\mathrm{u}$ / : dans la séquence « d'habitude ».

\subsubsection{Locutrice 6}

Figure 17. - Valeurs formantiques brutes $(\mathrm{Hz})$ : phases I et II.

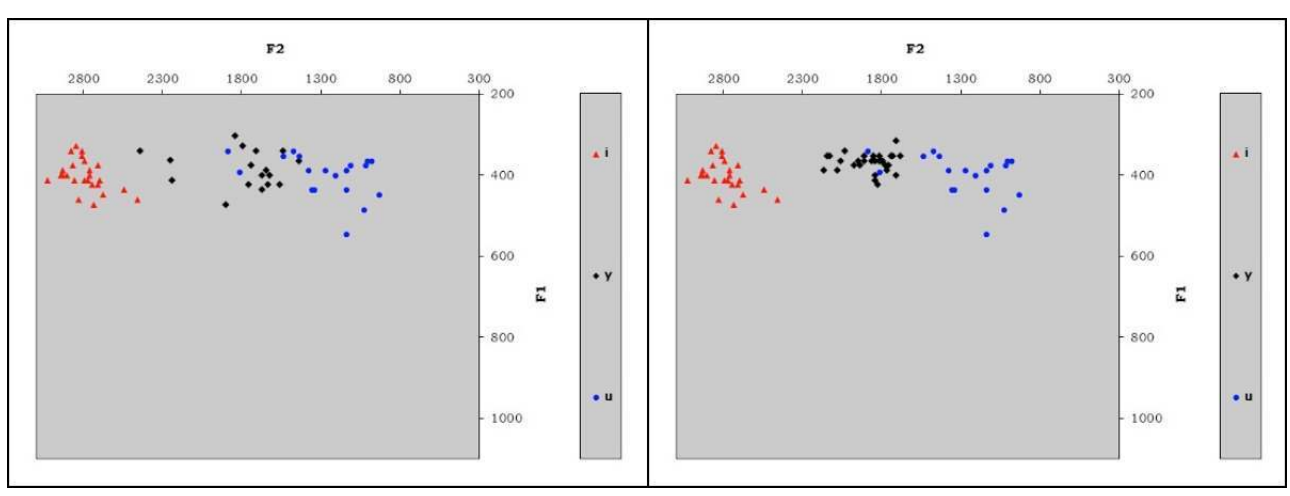

Figure 18. - Valeurs formantiques brutes $(\mathrm{Hz})$ : phase III.

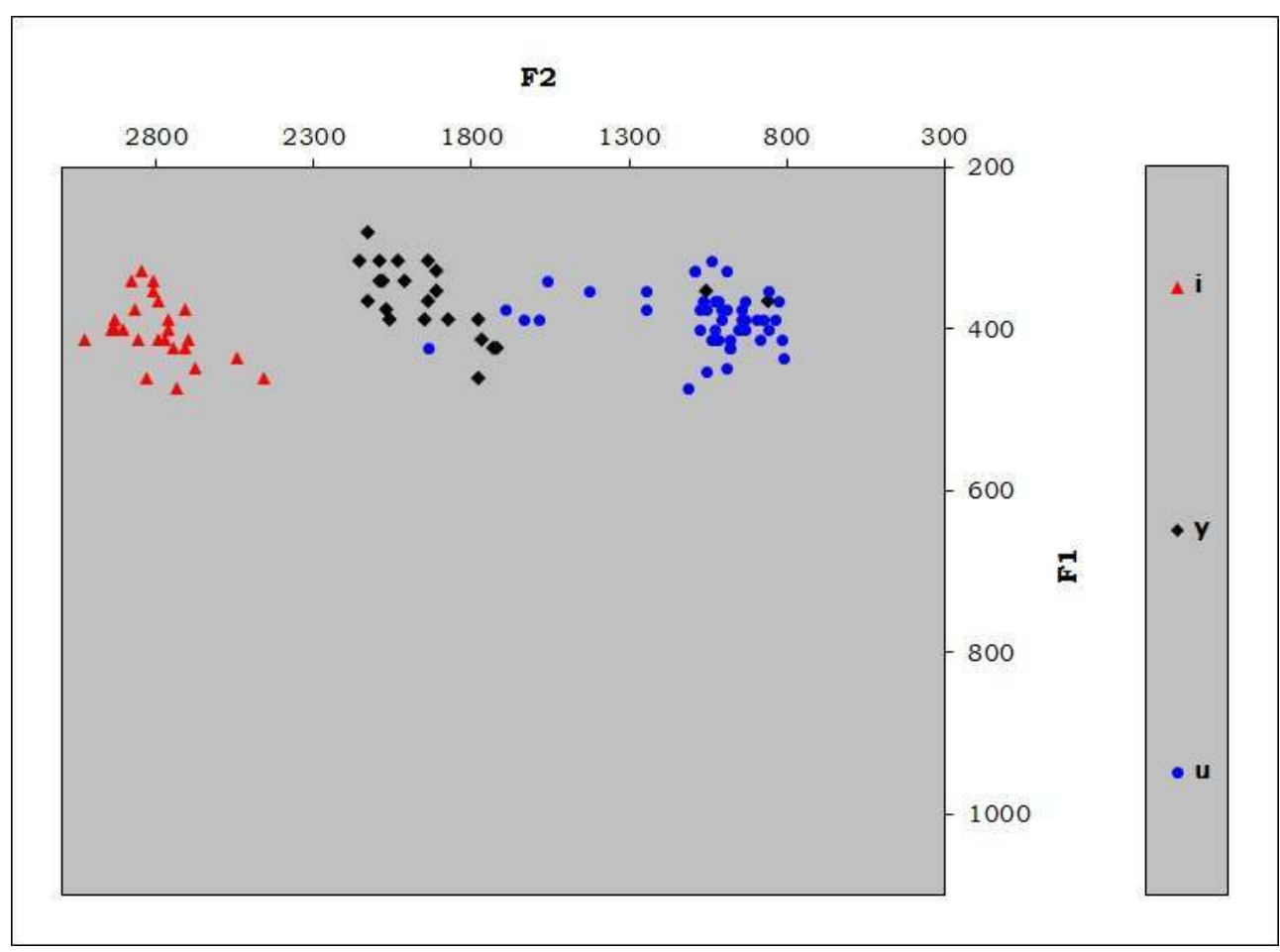

En phase I, le diagnostic est le même que précédemment : une confusion des /y/ et des / $\mathrm{u} /$ dans une zone qui s'étend entre 1379 et $1886 \mathrm{~Hz}$. Nous avons décidé de la faire travailler sur la remédiation $\mathrm{n}^{\circ} 1$ (voir fig. 4, éclaircissement) afin de séparer les /y/ et / $\mathrm{u} /$ et de la faire travailler en phase III également sur le $/ \mathrm{u} /$.

La phase de répétition des stimuli a très bien fonctionné, l'aire du /y/ est bien placée et elle s'est resserrée. En phase III, on observe une légère régression : le /y/ semble acquis 
malgré deux occurrences erronées prononcées / $\mathrm{u}$ / (dans les mots «vendu » et « vu»), mais le / $\mathrm{u} /$ est affecté en retour. Six occurrences tendent à l'antériorisation pour le / $\mathrm{u} /$ et ce phénomène a déjà été observé ici et dans notre précédente étude comparative (Hamm \& Dańko, 2017).

\subsection{Synthèse et discussion autour des résultats obtenus}

41 Nous avons prouvé par la juxtaposition de nos résultats qu'un contexte de production optimal permet un déplacement de l'aire des / $y /$ très manifeste pour tous nos locuteurs. En l'absence d'un groupe test, il n'est néanmoins pas possible d'imputer cette progression à la phase de remédiation seule. Certes, nos contextes de production étaient différents (consonnes subséquentes notamment) et non contrôlés, notre objectif dans le cadre de cette étude n'étant pas d'analyser le degré d'efficacité de chaque procédé relevant de la MVT utilisé séparément (intonation, entourage consonantique facilitant). Vu le nombre restreint d'occurrences à l'étude, nous n'avons pas distingué les conditions de production : lecture à haute voix, narration guidée, narration semi-spontanée. Il serait effectivement souhaitable de vérifier quelle condition serait la plus favorable à la correction phonétique en fonction des prédispositions de chaque apprenant.

Nos corpus sont courts, des biais pouvant ainsi être provoqués, et la focalisation de l'attention des étudiants sur la prononciation $\mathrm{du} / \mathrm{y} /$ au niveau de la deuxième phase et plus encore au niveau de la troisième phase de l'expérimentation était plus élevée.

De même, une représentation triangulaire F1/F2 n'englobe pas toute la réalité articulatoire, acoustique, perceptive, psychologique ou cognitive. Néanmoins, après un mois d'entrainement selon les principes de la méthode verbo-tonale d'intégration phonétique (intervention directement insitu et dans l'émission $\mathrm{du}$ message, d'entrainement à la maison sur le petit corpus de la phase II donné à chaque locuteur selon sa tendance à l'erreur), les progrès ont été conséquents: l'analyse acoustique prouve que les trois voyelles de petite aperture du français, /i, y, u/, peuvent être bien mises en place. En l'absence d'un groupe test, nous ne pouvons pas imputer cette progression à la phase d'entrainement seule, mais il est serait tout à fait possible d'obtenir une prononciation quasi authentique en peu de temps à condition de s'en donner les moyens.

Il nous resterait ultérieurement à enregistrer et à analyser les productions de nos apprenants en dehors d'une salle de classe dans le cadre d'une conversation spontanée et non plus semi-spontanée ou dirigée. La bi-focalisation sur le sens et sur la forme baisse en général le niveau de concentration sur la qualité de la prononciation chez les apprenants débutants.

\section{Conclusion et perspectives}

Même si nos résultats sont prometteurs, nous ne pouvons pas généraliser pour l'instant, car nous ignorons encore de quelle manière s'opère le transfert en dehors d'une salle de classe. Dégager le champ perceptivo-moteur de l'apprenant, permettre une perception lui garantissant une production optimale du/y/ qui sera rendue au fur et à mesure quasi inconsciente, pourraient pourtant être le moyen d'accès à toutes les autres compétences, linguistiques et communicatives. 


\section{(fonction physiologique de l'oreille). Cela consisterait à effectuer des filtrages et donc à modifier directement le signal sonore sur un seul phonème par exemple. \\ souhaiterions également proposer ultérieurement à l'usage du public polonophone, en réponse à des difficultés réelles bien définies, un manuel d'apprentissage des sons du français baignés dans leur moule prosodique, au sein d'actes de parole et au travers de situations de communication concrètes, en exploitant l'apport de l'approche verbo- tonale. Ce manuel sera fondé sur les résultats des études les plus récentes sur la perception des sons du français par le public polonais, les résultats des études acoustiques contrastives (français-polonais) qui permettent de définir/confirmer les difficultés de prononciation réelles, spécifiques de ce public, et les résultats de nos études acoustiques qui prouvent l'utilité des solutions apportées par l'approche verbo-tonale.}

l'hypothèse du filtre phonologique (fonction cérébrale, cognitive) et le filtrage acoustique

\section{BIBLIOGRAPHIE}

BEST, Catherine T. (1994). The Emergence of Native-Language Phonological Influences in Infants: A Perceptual Assimilation Model. Dans J. C. Goodman \& H. C. Nusbaum (dir.), The Development of Speech Perception: The Transition from Speech Sounds to Spoken Words (p. 167-224). Cambridge : MIT Press.

BILLEREY, Bożena. (2004). La perception des phonèmes français chez les apprenants polonais. Élaboration d'un outil pédagogique en FLE dans l'optique verbo-tonale de correction phonétique (Thèse de doctorat en sciences du langage). Lille : ANRT.

BILLIÈRES, Michel. (2005). Les pratiques du verbo-tonal. Retour aux sources. Dans M. Berré (dir.), Linguistique de la parole et apprentissage des langues. Questions autour de la méthode verbo-tonale de P. Guberina (p. 67-87). Mons : CIPA.

Boersma, Paul \& WeEnink, David. (2010). Praat: Doing Phonetics by Computer (version 5.1.29). Disponible en ligne sur $<$ www.praat.org $>$.

Callamand, Monique. (1981). Méthodologie de l'enseignement de la prononciation. Paris : Clé International.

DAŃKO, Magdalena, SAUVAGE, Jérémi \& HIRSCH, Fabrice. (2015). La perception phonémique en français des apprenants polonophones. L'Information grammaticale, 146, 32-38.

DŁUSKA, Maria. (1950/1983). Fonetyka polska. Artykulacje głosek polskich. Varsovie, Cracovie : Państwowe Wydawnictwo Naukowe.

FAURÉ, Georges \& DI CRISTO, Albert. (1977). Le français par le dialogue. Paris : Hachette.

FLEGE, James Emil. (1995). Second Language Speech Learning: Theory, Findings and Problems. Dans W. Strange (dir.), Speech Perception and Linguistic Experience: Theoretical and Methodological Issues in Cross-Language Speech Research (p. 233-277). Baltimore : York Press. 
Gajos, Mieczysław. (2010). Podsystemy jĘzyka w praktyce glottodydaktycznej. Fonetyka. Łódź :

Wydawnictwo Uniwersytetu Łódzkiego.

GEORGETON, Lauriane, PAILLEREAU, Nikola, LANDRON, Simon, GAO, Jiayin \& KAMIYAMA, Takeki. (2012). Analyse formantique des voyelles orales du français en contexte isolé : à la recherche d'une référence pour les apprenants de FLE. Dans Actes de la conférence conjointe JEP-TALN-RECITAL 1 (p. 145-152). Grenoble.

GUBERINA, Petar. (1971). Introduction à la méthode verbo-tonale de correction phonétique. Paris : Didier Érudition.

GuBERINA, Petar. (2003). Rétrospection. Zagreb : Ar Tresor Naklada.

GuIMBRETIÈRE, Élisabeth. (1996). Phonétique et enseignement de l'oral. Paris : Didier.

HAMm, Dominique \& DAŃKO, Magdalena. (2017). Perception et traque de « l'accent étranger » : la production des voyelles orales en FLE par des polonophones. Écho des études romanes, XIII(1), 101-122.

INTRAVAIA, Pietro. (2000). Formation des professeurs de langue en phonétique corrective. Le système verbo-tonal. Paris : Didier Érudition.

KuHL, Patricia K. (1991). Human Adults and Human Infants Show a "Perceptual Magnet Effect" for the Prototypes of Speech Categories, Monkeys Do Not. Perception and Psychophysics, 50(2), 93-107.

LADO, Robert. (1957). Linguistics across Cultures. Ann Arbor : University of Michigan Press.

LHOTE, Élisabeth. (1995). Enseigner l'oral en interaction. Percevoir, écouter, comprendre. Paris :

Hachette.

LHOTE, Élisabeth. (1990). Le paysage sonore d'une langue, le français. Hambourg : H. Buske.

MACIOŁEK, Marcin \& TAMBoR, Jolanta. (2012). Głoski polskie. Katowice : Wydawnictwo Gnom.

MACKEY, William F. (1976). Bilinguisme et contact des langues. Paris : Klincksieck.

OSTASZEWSKA, Danuta \& TAMBOR, Jolanta. (2006). Fonetyka i fonologia wspótczesnego języka polskiego. Varsovie : PWN.

Polivanov, Evgueni. (1931). La perception des sons d'une langue étrangère. Travaux du Cercle de linguistique de Prague, 4, 79-96.

RENARD, Raymond. (1974). Introduction à la méthode verbo-tonale de correction phonétique. Mayenne : Didier.

TRoubetzKoy, Nikolaï Sergueïevitch. (1939/2005). Principes de phonologie. Paris : Klincksieck.

EsSEN, Otto von. (1967). Fonetyka ogólna i stosowana. Varsovie : Państwowe Wydawnictwo Naukowe. Wioland, François. (2005). La vie sociale des sons du français. Paris : L'Harmattan.

WIERZCHOWSKA, Bożena. (1980). Fonetyka i fonologia jĘzyka polskiego. Wrocław, Varsovie, Cracovie, Gdańsk : Zakład Narodowy imienia Ossolińskich Wydawnictwo Polskiej Akademii Nauk.

\section{RÉSUMÉS}

Notre postulat de base est que toute voix nait d'une écoute. Nous nous focaliserons sur les difficultés de prononciation de la voyelle /y/ par des apprenants polonophones de niveau A1/A2 : une étude descriptive et comparative préliminaire, corroborée par une étude acoustique, a 
permis de déterminer que le phonème /y/ est souvent prononcé : 1) trop postérieur et/ou trop relâché (tendant ainsi vers le $/ \mathrm{u} /$ ) ; 2 ) trop antérieur (tendant vers le /i/). Or, les voyelles /i/ et / $\mathrm{u} /$ existent en polonais et sont les sons correspondants les plus proches de la voyelle /y/, absente dans le système vocalique polonais. Cette étude acoustique se fonde sur trois phases d'enregistrement/analyse de données : I) phase diagnostique permettant de déceler la tendance de chaque locuteur sur l'axe de l'acuité ; II) phase de répétition de stimuli selon les principes de la méthode verbo-tonale d'intégration phonétique (MVT); III) phase de réinvestissement des compétences à l'issue d'un mois d'entrainement par la méthode verbo-tonale à l'université d'Opole (Pologne).

Our basic assumption is that all voices are born from listening. We will focus on the pronouncing difficulties of the vowel/y/ by Polish learners of level A1/A2: a preliminary descriptive and comparative study, corroborated by an acoustic study, determined that the phoneme /y/ is often pronounced: 1) too posterior and/or too loose (thus tending towards the $/ \mathrm{u} /$ ); 2) too anterior (tending towards the /i/). However, the vowels /i/ and / $\mathrm{u} /$ exist in Polish and are the closest corresponding sounds to the vowel / $y /$, absent in the Polish vocal system. This acoustic study is based on three phases of data recording/analysis: I) diagnostic phase-to detect the tendency of each speaker on the axis of acuity; II) phase of repetition of stimuli according to the principles of the verbo-tonal method of phonetic integration (MVT); III) phase of reinvestment of the skills at the end of a month of corrective phonetics class by the verbo-tonal method at the University of Opole (Poland).

\section{INDEX}

Keywords : Polish-speaking learners, didactics of oral language, perception, spoken French and French as a Foreign Language, contrastive and corrective phonetics, interlanguage

Mots-clés : apprenants polonais, didactique de l'oral, perception, français parlé et FLE, phonétique contrastive et corrective, interlangue

\section{AUTEURS}

\section{DOMINIQUE HAMM}

Institut de phonétique de Strasbourg (IPS), Université de Strasbourg, France

\section{MAGDALENA DAŃKO}

Institut de culture et langue françaises, Université d'Opole (UO), Pologne 